\title{
A PC-based System for Long-term Monitoring of Animal Activity
}

\author{
B. M. Wu ${ }^{1}$, F. H.Y. Chan ${ }^{1}$, F. K. Lam ${ }^{1}$, M. C. Lam ${ }^{1}$, Paul W. F. Poon ${ }^{2}$, A. M. S. Poon ${ }^{3}$ \\ ${ }^{1}$ Department of Electrical and Electronic Engineering, The University of Hong Kong, Hong Kong \\ ${ }^{2}$ Department of Physiology, School of Medicine, National Cheng Kung University, Tainan, Taiwan \\ ${ }^{3}$ Department of Physiology, The University of Hong Kong, Hong Kong \\ Email: fhychan@eee.hku.hk
}

\begin{abstract}
This paper describes a PC-based animal locomotor and sound activities synchronous analysis and recording system. In the former, using video recording and image analysis techniques, the geometric locations of an animal in a cage and its bodily displacement areas between consecutive time in two-dimensions were detected. Tremendous data reduction rate has also been obtained (512x512:4), which facilitates our PC computer (Pentium 100) to perform a long-term (up to several weeks according to the space of hard disk) and on-line (1 sec) analysis and storage of the animal locomotor signals. In the latter, the sounds generated by the animal were recorded at the cage over a consecutive 1-sec time and its root mean square (RMS) value was used to index the sound level. Our preliminary study showed that such a combination of monitoring and recording system gives a faithful and comprehensive representation of animal activity.
\end{abstract}

Index Terms - Locomotion, Displacement area, Location, Sound, Image analysis, Long-term monitoring

\section{INTRODUCTION}

In the biomedical field, it is frequently necessary to measure animals or human behavior in a quantitative way. This is particularly important in the study of neuroscience, behavioral physiology or psychopharmacology. For animal activity analysis, several devices are now available to detect the location of the animal; these include photocells [1], switches [2], video camera [3] and other devices. However, since all such devices commonly used the distance moved by the animal between adjunct time to index the intensity of animal activity, only large bodily movements can be detected. The distance index is less sensitive to those vigorous but fine movements like scratching, self-rotation, drinking and feeding. In order to facilitate the monitoring of these small animal locomotor activities, a multi-parameter combining monitoring approach was proposed in this study. Besides measuring the location, we complementarily recorded the bodily displacement areas of the animal between consecutive time and the sounds generated by the animal. In comparison to the distance index, the displacement area index gives a more sensitive measurement of animal movements due to its pixel recording that will be described in details in the later part of this paper. The reason for sound recording is based on the fact that animals generate sounds even without moving their bodies. We believe that a simultaneous monitoring of the location, the bodily displacement area, and the sound will reflect more comprehensively the animal behavior. A system was developed with the location and the displacement area detected by incorporating video recording with image analysis techniques. Those fine movements like chewing, feeding and drinking which involved little lateral body movements were monitored by recording the acoustic level of the sound emitted by the animal. There are various features in both hardware setups and software implementation to achieve a long-term and on-line monitoring and recording of the animal activity. This paper mainly presents hardware setup and software features of the system. Some preliminary results are presented.

\section{HARDWARE SETUP}

The whole system is shown schematically in Fig.1. A small animal (mouse) was housed in a top-opened perspex cage placed in a dedicated sound- and light-shielded chamber (Industrial Acoustic Corporation, L240 x W180 x H220 in $\mathrm{cm}$ ) equipped with ventilation and light control (light-dark cycle). The cage was viewed by a low light working ( 0.1 lux) video camera (Pulnix TM560) mounted on the ceiling of the chamber 2 meters above the cage. The video signal was digitized by an 8-bit image grabber (DT2851) in real-time $(25$ frames $/ \mathrm{sec}$ ) and with a spatial resolution of $512 \times 512$ pixels. It was also sent to a video monitor (National-45) for observation of the experiment and to a video cassette tape recorder (VCTR, Mitsubishi-B31) for off-line confirmation of the actual activity task of the animal. At the same time, a microphone (B\&K 4149), which was placed near the cage $(10 \mathrm{~cm})$, picked up sound generated by the activity of the animal. The sound signal was then amplified (PARC113) and digitized at $20 \mathrm{kHz}$ with a 12-bit signal acquisition board (DT2801A). The light-dark cycle in the chamber was controlled by a light schedule controller (Timer-1) synchronized to the time of the computer. The day time was simulated by white light of intensity 195 lux illuminating from 4 energy saving bulbs placed at 4 corners of the cage. 
The night time was simulated by a 7.5 lux red light illuminating from 2 energy saving bulbs with red filter located at two sides of the cage. Two low-noise ventilation systems were used to provide continuous flow of air in the chamber. To provide a large contrast between the animal and the cage background, the bottom of cage was painted to white when black animals such as mice were studied. Also, to further minimize interference, the VCTR, the video monitor and the microcomputer were all located outside the chamber. This arrangement also facilitates observation of the researcher. Finally, by adjusting the zoom, iris and focus of the camera, good contrast video images that just view the whole field of the cage were obtained, and a control background acoustic level down to $20 \mathrm{~dB}$ SPL achieved inside the chamber.

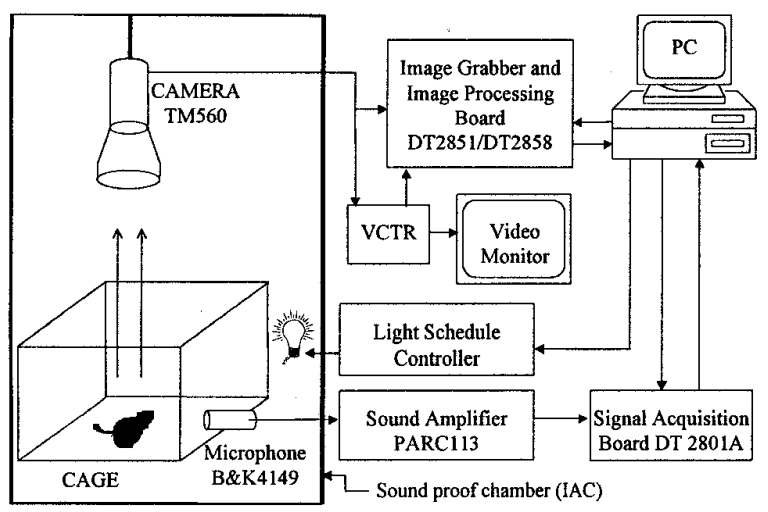

Fig.1. Schematic of the activity monitoring system

\section{SOFTWARE}

With proper settings, it is easy for the computer to identify the animal from the background of the cage and detect its location, displacement area, and detect the sound emitted by the animal. A program was developed which performs a long-term on-line analysis and recording of the animal activity using hardware features of the image grabber and the signal acquisition board. An off-line data processing program was also developed. It provides a variety of functions to view, search and save the acquired animal activity signals at different time resolutions. All on-line acquisition and off-line processing programs were written in Visual $\mathrm{C}++5.0$.

\section{A. On-line Data Processing}

\section{1) Locomotor Activity Monitoring}

\section{Thresholding of Image}

in our study, each frame of $512 \times 512$ 8-bit image in theory needs $256 \mathrm{k}$ bytes storage space. As a result, a longterm monitoring will need a large amount of storage space (e.g., more that $150 \mathrm{G}$ bytes / per week using a $1 \mathrm{~Hz}$ sampling rate). Data compression technique was then adopted to achieve a long-term and on-line analysis and storage of the animal activity signals. On the other hand, with proper settings in the system hardware, satisfactory image contrast between the animal and the background was obtained. The gray level values of the animal and the background always separate bimodally in the histogram, showing two main peaks representing the animal and the background of the cage respectively. Image thresholding technique was thus adopted. Using single threshold method, which reduces the complexity of the multi-level frame into a two-level picture, we separated the animal from the cage background. Different thresholding values were used during the light and dark phases (interactively specified prior to the data acquisition). Once the threshold was determined, all the pixels with gray level below $T$ were set to 0 and all gray level values larger than $T$ were set to 255 , the multi-level locomotion image was reduced into a two-level picture. Thus the images became all white with only the mice being black (Fig. 2 A, B).

\section{Detection of Animal Bodily Displacement}

Following the thresholding procedure, the bodily displacements of the animal across consecutive binary images were detected by a subtracting-counting procedure. For each new image (Fig. 2B), a difference image (Fig. 2C) was first computed by subtracting the previous image (Fig. 2A) from the current image pixel by pixel. Then, the number of both positive and negative pixels was counted. This counted value was in proportion to the displacement area of the animal body across two images. When the animal does not move, it is close to zero. When the animal produces a large bodily movement such that there is no overlap between two animal bodies in two consecutive images, it has a maximum value equal to the summation of areas of two animal bodies. Therefore, the displacement area could be taken as an index of the locomotor activity. Furthermore, by using sufficiently high sampling rate, the displacement could be limited not exceeding this maximum value. Suppose the areas of the animal images are equal to $A_{t}$ and $A_{t+1}$ at time $t$ and $t+1$, respectively, in this study, the displacement of the animal during the neighboring time $t$ and $t+l$ was defined as

$$
D_{A}=\left|\left(A_{t+1}-A_{t}\right)\right| / 2 \text {. }
$$

The time series of the displacement area formed the locomotor signal (lower panel of Fig. 2). In this way, each difference image was reduced to a single number, only 4bytes of memory were sufficient to store the result (as opposed to $512 \times 512$ bytes images). This drastic reduction in data size (from $512 \times 512$ to 4 per image) facilitated our PC computer to long-term and on-line store and analyze the locomotor signal derived from a large quantity of frames (e.g. $\mathrm{n}=86400 /$ day), and thus overcomes the large memory requirements for long-term animal behavior monitoring. 


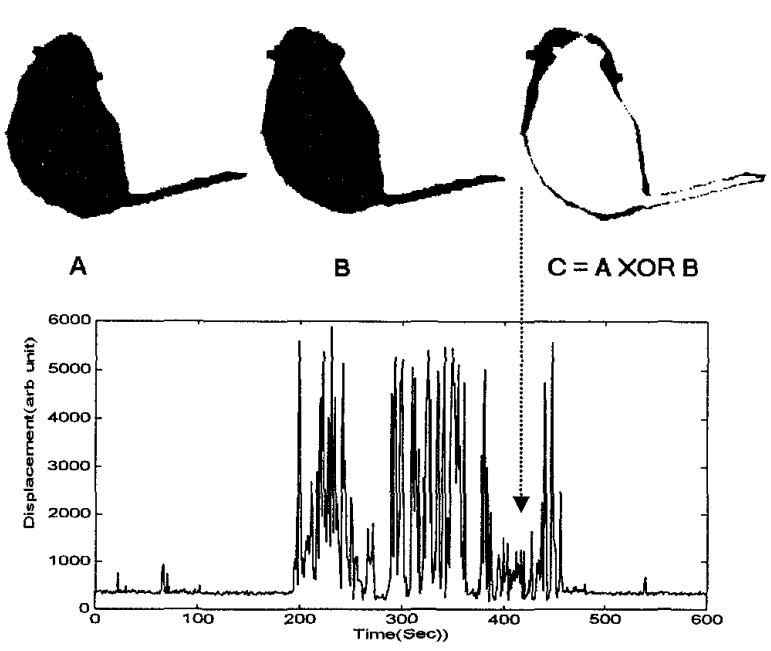

Fig.2. Illustration of the generation of the gross locomotor activity index

\section{Detection of Animal Location}

Since the animal is the largest object in the cage, it is easy to identify the animal from the background according to its horizontal and vertical dimensions. A fast searching procedure was developed. First, we searched the animal following a coarse to fine strategy. That is, a larger step in both horizontal and vertical directions was first used which quickly locates the animal in the cage. Once the animal was found, a fine step was used to localize the animal more accurately. There are different ways to define the location of an animal. Some required putting a small marker on the animal; others calculated the centroid of the animal. In this study, we presented a rectangle representation by extracting a rectangle, which just enclosed the animal body. Before the fine searching was carried out, one or two twice open (first erosion then dilation) operations were carried out to reduce the effect of mouse's tail on determination of the rectangle. Finally, we simply calculated the center of the rectangle as the location index of the animal. To further facilitate the searching speed, the frame-correlation between the animal movement was also used, we always started each searching procedure at the animal's previous location, because it is often the case that the animal would not move drastically from previous frame.

\section{2) Sound Activity Recording}

For animal sound monitoring, using the signal acquisition board DT2801A, we continuously digitized the acoustic signal recorded at the cage at a sampled at a rate of $20 \mathrm{kHz}$. Then we calculated the root-mean-square (RMS) value of the sound signals over each consecutive time window, of $1 \mathrm{sec}$ and used it as an index to reflect the sound activity level.

\section{3) Implementation of the Program}

Following the strategies described above, we implemented the program on a Pentium 100 PC by using hardware features of the image grabber and the signal acquisition board, giving a long-term and on-line analysis and recording of the animal activity. Two on-board functions of the DT2851 image series product are very useful for our study. (1) It has an input Look Up Table (LUT) which can be configured to fast implement simple image gray transforms such as gamma calibration, image enhancement, and image thresholding. (2) The auxiliary processing board (DT2858) can implement on-line logic operations such as OR, AND, $\mathrm{XOR}$, and mathematical operations such as ADD and SUBTRACTION as well as statistical processing like histogram calculation. In this study, we implemented the thresholding operation by updating the input LUT of the image grabber DT2851. Since the image has been set into two-levels, we performed the absolute-subtraction operation using the on-board XOR operation. We also implemented the counting operation using DT2851's on-board fast histogram calculation. For sound recording, to have a non-stop acquisition, an alternatively working model was developed. DT2801A's two $64 \mathrm{~K}$ memory blocks sampled alternatively and the sound signals transferred during the consecutive time window into the computer for RMS calculation. Finally, the displacement area, rectangle coordination of the animal and the RMS sound values were detected on-line and stored on harddisk at a rate of $1 \mathrm{~Hz}$, which is sufficient to detect fast changes in body position of a mouse (usually not exceeding one body dimension in excursion). A flow chart of the online data acquisition program is shown in Fig. 3.

\section{B. Off-line Data Processing}

An off-line processing program was developed which can view, search, and save the activity signals at different time resolutions. Particularly, since the time cues have also been saved, it can also correspond with the recorded data with the recorded tape images.

\section{RESULTS}

In order to assess the capability of the system for behavioral study, a preliminary study was carried out. We used this system to study temporal patterns and spatial distribution of spontaneous activity of a mouse under normal light-dark condition ( $12 \mathrm{hr}$ light: $12 \mathrm{hr}$ dark). Some preliminary results were obtained. Fig.4 gives an overall location map (upper panel) and a combined displacement area-sound plot (lower panel). It is noticed that the animal preferred to stay mainly at boundaries and corners of the cage, while spending nearly no time in the middle of the cage. There are also two preferred areas (eclipses) located around the feeding and drinking sites (arrows). The time profiles of displacement area and sound illustrate temporal patterns of the animal activity. In particular, several types of 
activity, such as bodily movements, drinking, eating and rest were identified by comparing with the recorded tape images. It can clearly be seen that there are different signal characteristics in both displacement and sound corresponding to different types of activities. For example, a continuous drinking is accompanied with no bodily movement. In theory, the animal's behavior can be recognized by a simultaneous analysis of the displacement area and sound signals together with the animal location instead of reading the tape.

\section{CONCLUSION AND DISCUSSION}

In this paper, we have developed a comprehensive PCbased animal activity monitoring system, which features a long-term, on-line, and simultaneous analysis and recording of locomotor and sound activities. Our preliminary study results have also confirmed the capability of the system for behavioral study in animals. Location mapping is useful to determine the animal trail and then distance, speed and distribution in the cage. The major advantage of using displacement area is its high sensitivity to small movements like scratching, self-rotation, drinking, feeding, and even breathing while sleeping. Sound monitoring can provide complementary activity information without major bodily movements. Both displacement and sound signals provide valuable temporal pattern information of the animal activity. More importantly, it allows us to identify animal behaviors instead of using complicated pattern recognition technique or interactively viewing the video tape. Such a combining monitoring strategy has facilitated complete study on animal behaviors. Data compression technique adopted has greatly simplified the task of data processing and has also saved system memory, and thus allowed long-term and on-line measurement and finally was able to implement the system on a PC. It is also low cost, and affordable.

The system could be widely used to study animal behavior in the study of neuroscience, behavioral physiology or psychopharmacology. But it should be noted that in this study we have focused our study on the two-dimensional movements of the animal, the vertical movements were ignored. For a more complete description, a 3D monitoring system may be needed using multi-camera systems.

\section{REFERENCES}

[1] Muramoto K., Ono, T., and Shibata R., A microcomputer based system for detailed analysis of rat behavior, Neuroscience Research, 1986, 4: 157-165.

[2] Ticher, A., Ashkenazi, I. E., Compound structure of rodents activity rhythm, Physiol. Behav., 1994, 57: 3740.

[3] Hoy J. B., Koehler P. G., Patterson R. S., A microcomputer-based system for real-time analysis of animal movement, J. Neurosci. Methods, 1996, Feb. 64(2): 157-61.

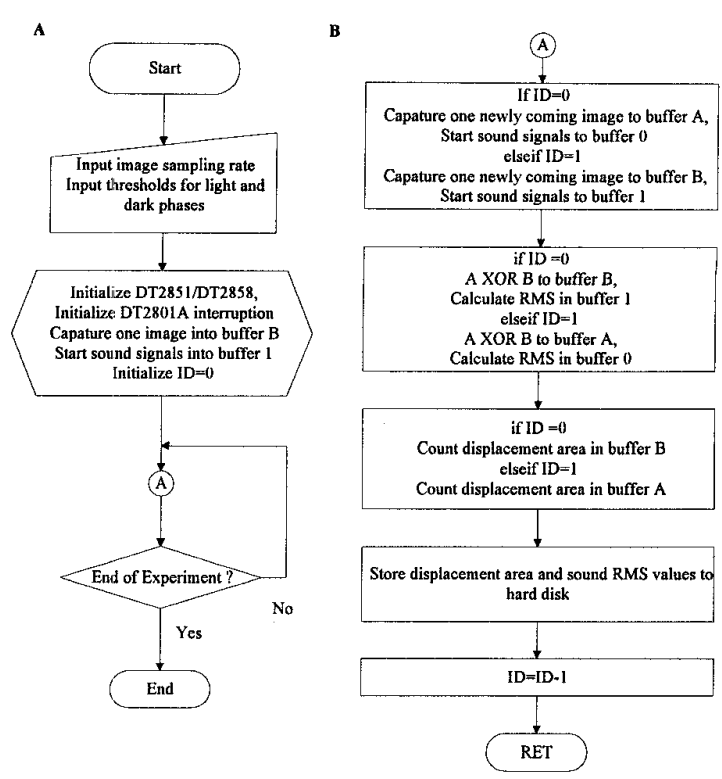

Fig.3. Flow chart of the on-line data acquisition program
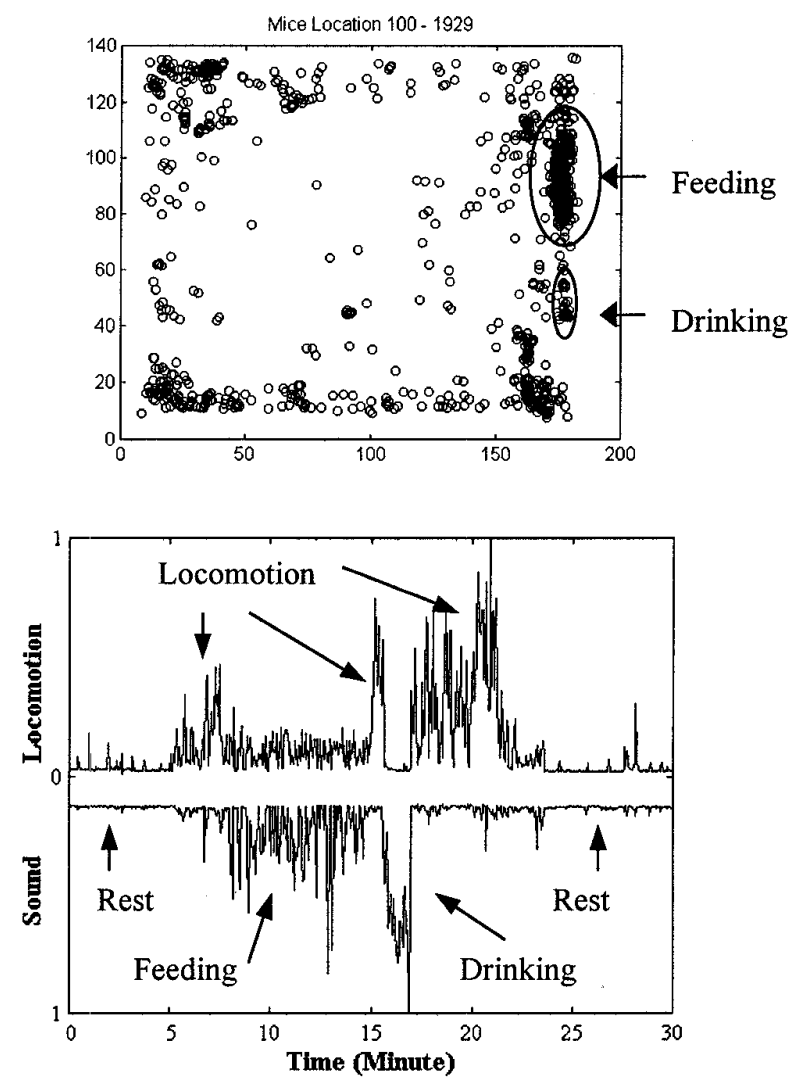

Fig.4. Location distribution (upper panel) and displacement area and sound activity profiles (lower panel) of a mouse over 30 minutes in the dark. 